\title{
Electron transport in crossed nanotubes with point contact
}

\author{
Viktor Margulis and Mikhail Pyataev* \\ Mordovian State University, Saransk, Russia
}

(Dated: December 18, 2018)

\begin{abstract}
The electron transport in a four-terminal nanodevice consisting of two crossed nanotubes is investigated in the framework of the Landauer-Buttiker formalism. The evident formula for the ballistic conductance of the device is found using a model of crossed conductive cylinders with a point contact between them. Sharp conductance dips stipulated by resonance scattering on the contact are shown to appear in the conductance of the first cylinder. The conductance between the cylinders has resonant behavior. The form and the position of resonant peaks are studied. Our results indicate that the form of asymmetric dips and peaks in the conductance differs from the well-known Fano line shape. We have shown that the maximal value of the conductance between cylinders does not exceed a unit of the conductance quantum.
\end{abstract}

PACS numbers: 73.63.Fg, 73.23.Ad, 73.63.Rt

\section{INTRODUCTION}

Carbon nanotubes are considered as a promising material for future nanoelectronic engineering due to their unique physical properties. The quantum coherent transport properties of carbon nanotubes have been confirmed by experimental results, indicating that the tube can be considered as a ballistic conductor at least up to $200 \mathrm{~nm} \cdot \underline{\underline{1}}$ Phase coherent transport and electron interference have been observed in single-walled ${ }^{2}$ and multi-walled ${ }^{3}$ carbon nanotubes. It was shown that superlattice properties of carbon nanotubes in a transverse electric field raise new possibilities for developing optoelectronic devices operating in the terahertz range of frequencies ${ }^{4}$. Recent theoretical studies have shown that electronic devices together with their metallic interconnects can, in principle, be fabricated on a single tube ${ }^{\frac{5}{}}$ Furthermore, nanotubes can operate under a variety of conditions and are compatible with many other materials and fabrication techniques. Several promising functional devices based on carbon nanotubes have been proposed $\underline{\underline{6}}^{-}$These devices include metallic wires,,$\underline{7}$ terahertz range emitters ${ }^{8}$ field effect transistors, $, 9,10,11,12,13$ and nanometer-size rectifying diodes $\underline{14,15,16,17}$

The design of the integrated electronic circuits implies the application of many contacted nanotubes. Therefore, transport properties of the contacts between nanotubes are of particular interest. In the last few years, electron transport in such contacts has been investigated experimentally $: 17,18,19,20$

A number of interesting theoretical models has been suggested to study physical properties of the contacts. ${ }^{21,22,23,24,25,26,27}$ The transmission through atom-contacted single-walled carbon nanotubes were calculated within the tight-binding approach $\stackrel{21}{1}^{\text {Multi- }}$ terminal junctions of single-walled carbon nanotubes were investigated using the classical molecular dynamics method $: 22$ Tight-binding calculations of the conductance of multiply connected metallic carbon nanotubes were carried out in Refs. 24,25. Parallel and crossed junctions of single-wall carbon nanotubes were studied in the framework of the tight-binding approximation ${ }^{23}$ Transport properties of three-terminal carbon nanotube junctions have been investigated within the scattering matrix approach. 27 The differential conductance of several crossed carbon nanotubes were calculated using the tight-binding model and the Green's function method ${ }^{26}$

It has been shown that the conductance through several multiterminal nanotube junctions exhibits Fano resonances $24,25,26,27$ This phenomenon emerges from the coherent interaction of a discrete state and a continuum and was first discovered by studying the asymmetric peak in helium spectrum ${ }^{28}$ Subsequent theoretical investigations have shown the occurrence of this effect in numerous mesoscopic devices, including quantum $\operatorname{dots}^{29,30}$ and quasi-one-dimensional channels with impurities $31,32,33,34$ Recently the resonances were observed experimentally in the ballistic conductance of a single-electron transistor ${ }^{35}$ and a quantum ring. 36 Asymmetric dips and peaks similar to the Fano resonances have been found in the conductance of carbon nanotubes $\stackrel{3,18,37}{=}$ The interest to this problem is stipulated by the possibility of application the phenomenon in high-sensitive resonant electronic devices. The resonances lead to large changes in current intensity in short intervals of voltage. This phenomenon may be used in designing of precise electronic devices.

The necessary condition for the emergence of Fano resonances is the existence of the discrete level in the continuous spectrum. In the case of carbon nanotube junctions, these discrete levels were attributed to pentagonal and heptagonal defects of the honeycomb lattice. ${ }^{24,25,26,27}$ However, the detailed atomic structure of the multiterminal nanotube junction still was not studied experimentally. Therefore, the origin of asymmetric line shapes in the conductance requires further theoretical investigations, especially in the case of multiwall carbon nanotubes.

It should be noted that the most of theoretical studies 
of the electron transport in the junctions were focused on nanotubes of sufficiently small diameters (about 1-4 nm). At the same time, the diameters of tubes used in some experiments ${ }^{18}$ were in the range of $25-30 \mathrm{~nm}$. The application of the tight-binding approach to this systems is somewhat difficult because it requires considerable amount of computer resources. Furthermore, physical meaning of the phenomenon is sometimes smeared in sufficiently accurate but very complicated models. Thus, it should be useful to study the electron transport through the contact between two nanotubes using a simple model which allows exact analytical solution. The simplest model with the geometry of a nanotube is a structureless two-dimensional cylindrical surface. Transport properties of the electron gas on the cylindrical surface have already been studied in the literature ${ }^{38,39,40,41,42}$ In particular, this model has been used for analysis of some electron properties of carbon nanotubes. $\stackrel{41,42}{ }$ Other interesting systems with cylindrical geometry are rolled GaAs/AlGaAs heterostructures. ${ }^{43,44}$

\section{HAMILTONIAN}

The purpose of the present paper is the theoretical investigation of the electron transport in a four-terminal nanodevice consisting of two crossed nanotubes with a point contact between them. Each tube is modeled by a conductive cylindrical surface of radius $r_{j}(j=1,2)$. The schematic view of the device is shown in Fig. 1.

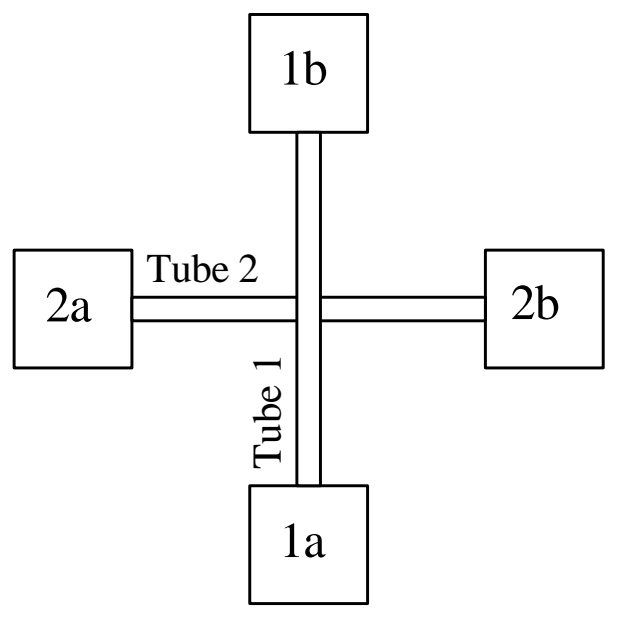

FIG. 1: Scheme of the device. Tube 1 lies over the tube 2 . Rectangles 1a,1b,2a, and 2b, represent electron reservoirs.

Our first goal is to construct the electron Hamiltonian of the system. If we ignore the contact between the cylinders, then the electronic states are described by the unperturbed Hamiltonian $H_{0}=H_{1} \oplus H_{2}$, where $H_{1}$ and $\mathrm{H}_{2}$ are electron Hamiltonians in the first and the second cylinder respectively. In this case, the electron wave function may be represented in the form of one-column matrix

$$
\psi=\left(\begin{array}{l}
\psi_{1} \\
\psi_{2}
\end{array}\right)
$$

Since the contact between cylinders is modeled by a single point, the Hamiltonian $H$ of the whole system is a point perturbation of the operator $H_{0}$. To obtain this perturbation we use the zero-range potential theory.

We introduce two independent cylindrical coordinate systems and denote the point on the cylinder by $\mathbf{q}=$ $(z, \varphi)$. Then the Hamiltonian $H_{j}$ has the form

$$
H_{j}=\frac{p_{z}^{2}}{2 m_{j}}+\frac{L_{z}^{2}}{2 m_{j} r_{j}^{2}},
$$

where $m_{j}$ is the electron effective mass in $j$-th nanotube, $p_{z}$ and $L_{z}$ are projections of the momentum and the angular momentum onto the axis of the cylinder. The electron effective mass depends on the size and chirality of the tube. For each cylinder, we use its own cylindrical coordinate system.

The spectrum of the Hamiltonian $H_{j}$ is given by the sum of the discrete part $E_{m}^{(j)}=\varepsilon_{j} m^{2}$ and the continuous part $p_{z}^{2} /\left(2 m_{j}\right)$

$$
E_{m, p_{z}}^{(j)}=\varepsilon_{j} m^{2}+p_{z}^{2} /\left(2 m_{j}\right),
$$

where $m$ is the magnetic quantum number and $\varepsilon_{j}=$ $\hbar^{2} /\left(2 m_{j} r_{j}^{2}\right)$. In the case of identical tubes $\left(r_{1}=r_{2}\right)$, we will omit below the subscript of $\varepsilon$.

To obtain the Hamiltonian $H$ of the whole system we have to define the point perturbation of the Hamiltonian $H_{0}$. For this purpose, we use linear boundary conditions at the point of the contact. Boundary values for the wave function $\psi_{j}$ of the electron in $j$-th cylinder are determined with the help of the zero-range potential theory $45,46,47$ The theory shows that the electron wave function $\psi_{j}(\mathbf{q})$ has the logarithmic singularity in a vicinity of the contact point $\mathbf{q}_{0}$

$$
\psi_{j}(\mathbf{q})=-u_{j} \ln \rho\left(\mathbf{q}, \mathbf{q}_{0}\right)+v_{j}+R(\mathbf{q}),
$$

where $\rho\left(\mathbf{q}, \mathbf{q}_{0}\right)$ is geodesic distance between the points $\mathbf{q}$ and $\mathbf{q}_{0}, u_{j}$ and $v_{j}$ are complex coefficients, and $R(\mathbf{q}) \rightarrow 0$ in the limit $\mathbf{q} \rightarrow \mathbf{q}_{0}$. The similar method has been used earlier in Refs. 48.49 .

It is clear that the boundary conditions at the point of contact are some linear relations between $u_{1}, v_{1}, u_{2}$, and $v_{2}$. The coefficients of the relations are not all independent ${ }^{47}$ since the Hamiltonian $H$ is Hermitian. Thus, the most general form of the boundary conditions is given by

$$
\left\{\begin{array}{l}
v_{1}-b_{1} u_{1}=a u_{2} \\
v_{2}-b_{2} u_{2}=a^{*} u_{1}
\end{array}\right.
$$

Here the coefficients $b_{1}$ and $b_{2}$ determine the strength of the zero-range potential at the point of contact and $a$ 
is a dimensionless parameter that is responsible for the coupling of the wave functions on different cylinders. According to the zero-range potential theory parameters $b_{j}$ can be represented in terms of scattering lengths $\lambda_{j}$ by the relation $b_{j}=2 \ln \lambda_{j}$. It should be noted that the zerorange potential is attractive and the strength of the potential decreases with increasing of $\lambda_{j}$. The limit $\lambda_{j} \rightarrow \infty$ corresponds to the absence of the point perturbation. We point out that the model of zero range potential is applicable when the size of the contact is much smaller than the Fermi wavelength of the electron.

\section{TRANSMISSION COEFFICIENTS}

In the paper, we investigate the conductance $G_{11}$ of the first cylinder and the conductance $G_{21}$ that is responsible for the electron transport from the first cylinder to the second one. According to the Landauer-Büttiker formula the zero-temperature conductance $G_{j i}$ can be expressed in terms of transmission coefficients $T_{m^{\prime} m}^{j i}$ from the state with magnetic quantum number $m$ in $i$-th cylinder to the state with $m^{\prime}$ in $j$-th cylinder

$$
G_{j i}=G_{0} \sum_{m^{\prime} m} T_{m^{\prime} m}^{j i} .
$$

Here $G_{0}=e^{2} / \pi \hbar$ is the conductance quantum and the sum is taken over all states with $E_{m} \leq \mu$, where $\mu$ is the Fermi energy.

The transmission coefficients are represented via transmission amplitudes $t_{m^{\prime} m}^{j i}$

$$
T_{m^{\prime} m}^{j i}=\frac{k_{m^{\prime}}^{(j)}}{k_{m}^{(i)}}\left|t_{m^{\prime} m}^{j i}\right|^{2} .
$$

To determine the amplitudes $t_{m^{\prime} m}^{j i}$ we need a solution of the Schrödinger equation for the Hamiltonian $H$. The zero-range potential theory allows us to represent the solution in terms of the Green function $G_{j}\left(\mathbf{q}, \mathbf{q}^{\prime}, E\right)$ for the operator $H_{j}$

$$
\left\{\begin{array}{l}
\psi_{1}(\mathbf{q})=\psi_{m}(\mathbf{q})+\alpha_{1} G_{1}\left(\mathbf{q}, \mathbf{q}_{0} ; E\right), \\
\psi_{2}(\mathbf{q})=\alpha_{2} G_{2}\left(\mathbf{q}, \mathbf{q}_{0} ; E\right),
\end{array}\right.
$$

where $\psi_{m}(\mathbf{q})=\exp \left(i k_{m} z+i m \varphi\right)$ is an incident wave, and coefficients $\alpha_{j}$ have to be determined from boundary conditions. The Green function $G_{j}\left(\mathbf{q}, \mathbf{q}^{\prime}, E\right)$ is given by ${ }^{49}$

$$
G_{j}\left(\mathbf{q}, \mathbf{q}^{\prime} ; E\right)=\frac{i m_{j}}{2 \pi \hbar^{2}} \sum_{m=-\infty}^{\infty} \frac{e^{i k_{m}^{(j)}\left|z-z^{\prime}\right|+i m\left(\varphi-\varphi^{\prime}\right)}}{k_{m}^{(j)} r},
$$

where $k_{m}^{(j)}=\sqrt{2 m_{j}\left(E-E_{m}^{(j)}\right)} / \hbar, \quad \operatorname{Re} k_{m} \geq 0$, and $\operatorname{Im} k_{m} \geq 0$.

Substituting the wave function (7) into Eq. (4), we obtain

$$
\left\{\begin{array}{l}
u_{j}=\frac{i m_{j}}{2 \pi \hbar^{2}} \alpha_{j}, \\
v_{j}=\delta_{j 1}+\alpha_{j} Q_{j}(E),
\end{array}\right.
$$

where $Q_{j}(E)$ is Krein's Q-function ${ }^{47}$ that is the renormalized Green function of the Hamiltonian $H_{j}$

$$
Q_{j}(E)=\lim _{\mathbf{q} \rightarrow \mathbf{q}_{0}}\left[\frac{2 \pi \hbar^{2}}{m_{j}} G_{j}\left(\mathbf{q}_{0}, \mathbf{q} ; E\right)+\ln \rho\left(\mathbf{q}_{0}, \mathbf{q}\right)\right] .
$$

To simplify our notations we denote $\widetilde{Q}_{j}(E)=Q_{j}(E)-b_{j}$. The explicit form of $\widetilde{Q}_{j}(E)$ was found in Ref. 49

$$
\widetilde{Q}_{j}(E)=\frac{i}{r_{j} k_{0}^{(j)}}+2 \sum_{m=1}^{\infty}\left(\frac{i}{r_{j} k_{m}^{(j)}}-\frac{1}{m}\right)+2 \ln \frac{r_{j}}{\lambda_{j}} .
$$

One can see from Eqs. (7) and (8) that the wave function $\psi_{j}(\mathbf{q})$ has the following asymptotics at $z \rightarrow \infty$ :

$$
\psi_{j}(\mathbf{q}) \simeq \sum_{m^{\prime}=m_{\min }}^{m_{\max }} t_{m^{\prime} m}^{j i} e^{i k_{m^{\prime}}^{(j)} z+i m^{\prime} \varphi},
$$

where $m_{\min }$ and $m_{\max }$ are minimal and maximal values of the magnetic quantum number for occupied states. The asymptotics of the wave function $\psi_{1}(\mathbf{q})$ in the first cylinder at $z \rightarrow-\infty$ is given by

$$
\psi_{1}(\mathbf{q}) \simeq e^{i k_{m}^{(1)} z+i m \varphi}+\sum_{m^{\prime}=m_{\min }}^{m_{\max }} r_{m^{\prime} m}^{11} e^{-i k_{m^{\prime}}^{(1)} z+i m^{\prime} \varphi},
$$

where $r_{m^{\prime} m}^{11}$ are reflection amplitudes. Elementary but cumbersome calculations show that the relation

$$
\sum_{m^{\prime}=m_{\min }}^{m_{\max }}\left[\frac{k_{m^{\prime}}^{(1)}}{k_{m}^{(1)}}\left(\left|r_{m^{\prime} m}^{11}\right|^{2}+\left|t_{m^{\prime} m}^{11}\right|^{2}\right)+2 \frac{k_{m^{\prime}}^{(2)}}{k_{m}^{(1)}}\left|t_{m^{\prime} m}^{21}\right|^{2}\right]=1
$$

is valid for an arbitrary energy $E$ that is the manifestation of the current conservation law for our system. Here factor 2 corresponds to equal probabilities for an electron to pass from lead $1 a$ to lead $2 a$ or $2 b$ (see Fig. (1).

Applying boundary conditions (5) to the wave function (77), we obtain the following form for the transmission amplitudes $t_{m^{\prime} m}^{11}$ :

$$
t_{m^{\prime} m}^{11}(E)=\delta_{m m^{\prime}}-\frac{i \widetilde{Q}_{2}}{\widetilde{Q}_{1} \widetilde{Q}_{2}-|a|^{2}} .
$$

The transmission coefficients $T_{m^{\prime} m}^{11}$ are given by

$$
T_{m^{\prime} m}^{11}(E)=\frac{k_{m^{\prime}}^{(1)}}{k_{m}^{(1)}}\left|\delta_{m m^{\prime}}-\frac{i \widetilde{Q}_{2}}{\widetilde{Q}_{1} \widetilde{Q}_{2}-|a|^{2}}\right|^{2} .
$$

We note that according to Eq. (10)

$$
\operatorname{Im} \widetilde{Q}_{j}=\sum_{m=m_{\min }}^{m_{\max }} \frac{1}{r_{j} k_{m}^{(j)}} .
$$

Thus, we can express the sum in Landauer's formula (6) in terms of the Q-function and obtain the following equation for the conductance $G_{11}(\mu)$ :

$$
\frac{G_{11}(\mu)}{G_{0}}=N(\mu)-\frac{\left(\operatorname{Im} \widetilde{Q}_{1}\right)^{2}\left|\widetilde{Q}_{2}\right|^{2}}{\left.\left.\left|\widetilde{Q}_{1} \widetilde{Q}_{2}-\right| a\right|^{2}\right|^{2}}-2 \frac{|a|^{2} \operatorname{Im} \widetilde{Q}_{1} \operatorname{Im} \widetilde{Q}_{2}}{\left.\left.\left|\widetilde{Q}_{1} \widetilde{Q}_{2}-\right| a\right|^{2}\right|^{2}} .
$$


Here $N(\mu)=m_{\max }-m_{\min }+1$ is the number of states with the energy smaller than $\mu$. It is worth mentioning that the possibility to represent the conductance in the explicit form is based on the application of zero-range potentials for modeling the contacts.

\section{RESULTS AND DISCUSSION}

Conductance $G_{11}$ as a function of the Fermi energy $\mu$ is represented in Figs. 22 5. If the contact between the cylinders is absent, then Eq. (15) contains only the first term, and the dependence $G_{11}(\mu)$ is step-like. The second term in Eq. (15) is responsible for the back-scattering on the contact point and the last term is stipulated by the transmission of electrons from the first nanotube to the second one.

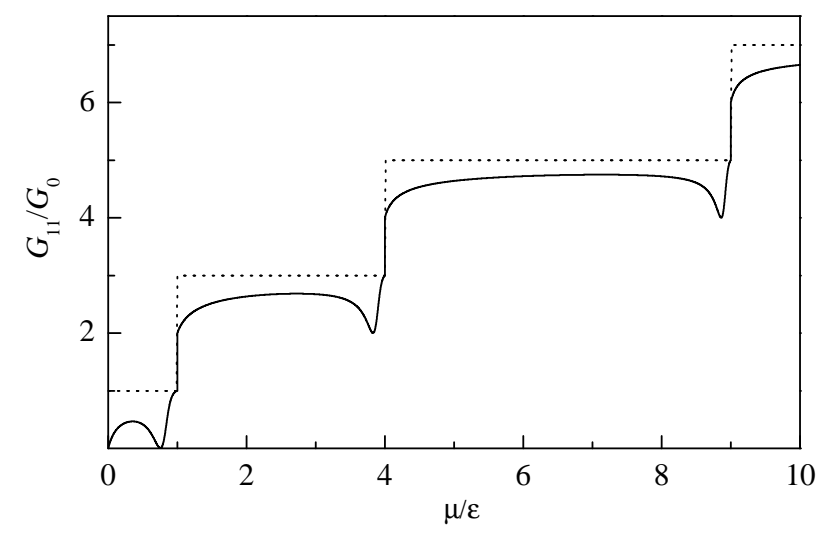

FIG. 2: Conductance $G_{11}$ versus the Fermi energy $\mu$ at $r_{1}=$ $r_{2}, \lambda_{1}=\lambda_{2}=3 r_{1}$, and $a=0.1$. Dotted line represents the conductance of the unperturbed cylinder.

At first, we consider the case of identical tubes $\left(r_{1}=r_{2}\right.$ and $\lambda_{1}=\lambda_{2}$ ). The presence of the zero-range perturbation at the point of contact leads to appearance of virtual levels $\tilde{E}=E_{R}-i \Gamma$ in the spectrum of the Hamiltonian $H$. Positions of the virtual levels are defined by equation

$$
\widetilde{Q}_{1}(\tilde{E}) \widetilde{Q}_{2}(\tilde{E})-|a|^{2}=0 .
$$

The scattering on the virtual levels leads to appearance of dips on the dependence $G_{11}(\mu)$. If the coupling between the wave functions on different cylinders is weak $(|a| \ll 1)$, then the virtual level $\tilde{E}$ is situated in the vicinity of the root $\tilde{E}_{1}$ of the equation $\widetilde{Q}_{1}\left(\tilde{E}_{1}\right)=0$. The conductance has only one dip on each plateau in this case. The dip is situated near the point $\operatorname{Re} \widetilde{Q}_{1}\left(E_{1}\right)=0$. If $\lambda_{1} \gg r_{1}$, then the dip is situated near the right edge of the conductance plateau. With decreasing $\lambda_{1}$, the dip shifts to lower energies and disappears reaching the left edge of the plateau. There are no dips of the conductance in the limit of the strong point perturbation $\left(\lambda_{j} \ll r_{j}\right)$ and the weak interaction between the wave

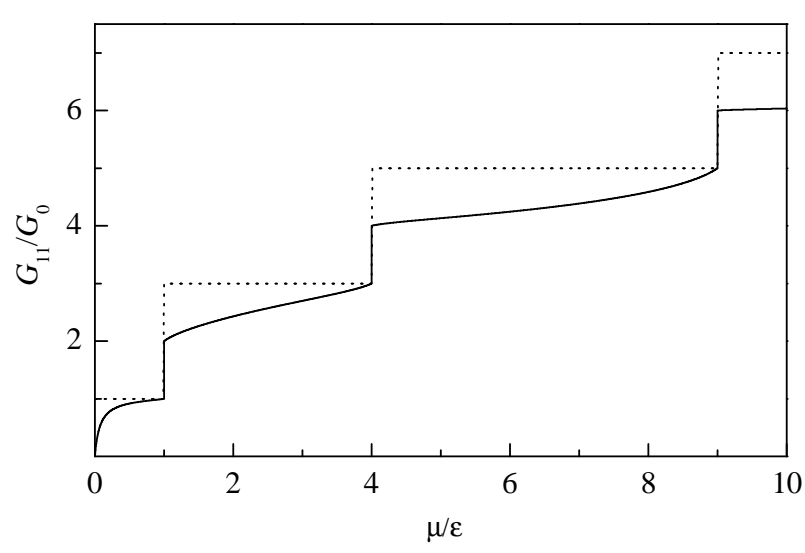

FIG. 3: Conductance $G_{11}$ versus the Fermi energy $\mu$ at $r_{1}=$ $r_{2}, \lambda_{1}=\lambda_{2}=0.15 r_{1}$, and $a=0.1$. As in the previous figure, dotted line represents the conductance of the unperturbed cylinder.

functions $(|a| \ll 1)$. The dependence $G_{11}(\mu)$ is monotonic under these conditions (Fig. 31).

If the coupling between the wave functions on different tubes is sufficiently strong $|a| \geq 1$, then additional conductance minima appear on the graph $G_{11}(\mu)$ (Fig. (4). These minima are stipulated by splitting of the virtual levels due to the interaction between electron states on different cylinders. In the case of the strong point perturbation $\left(\lambda_{j} \ll r_{j}\right)$ and the strong interaction between the wave functions $(|a| \gg 1)$, the dependence $G_{11}(\mu)$ contains one dip on each conductance plateau.

Let us determine the minimal values of the conductance $G_{11}(\mu)$. We denote $\xi_{j}=\operatorname{Re} \widetilde{Q}_{j} / \operatorname{Im} \widetilde{Q}_{j}$ and $\eta=$ $|a|^{2} /\left(\operatorname{Im} \widetilde{Q}_{1} \operatorname{Im} \widetilde{Q}_{2}\right)>0$. Then we can express the conductance $G_{21}(\mu)$ in terms of three real variables $\xi_{1}, \xi_{2}$ and $\eta$

$$
G_{11}(\mu)=G_{0}\left(N(\mu)-\frac{1}{1+f_{11}(\mu)}\right)
$$

where

$$
f_{11}(\mu)=\frac{\left(\xi_{1} \xi_{2}-\eta\right)^{2}+\xi_{1}^{2}}{1+\xi_{1}^{2}+2 \eta} \geq 0
$$

One can see from Eq. (17) that the depth of dips equals $G_{0}$ when $f_{11}=0$. That is possible only when the interaction between the tubes is absent $(a=0)$. Otherwise, the depth of dips is less than $G_{0}$.

If radii of nanotubes are different, then the dependence $G_{11}(\mu)$ contains additional peaks and dips in the vicinity of the points $E_{m}^{(2)}$. Similar effects occur in the case of different electron effective masses. To study the form of the curve $G_{11}(\mu)$ we consider the asymptotics of the Qfunction in the vicinity of $E_{m}^{(j)}$. One can see from Eq. (10) 


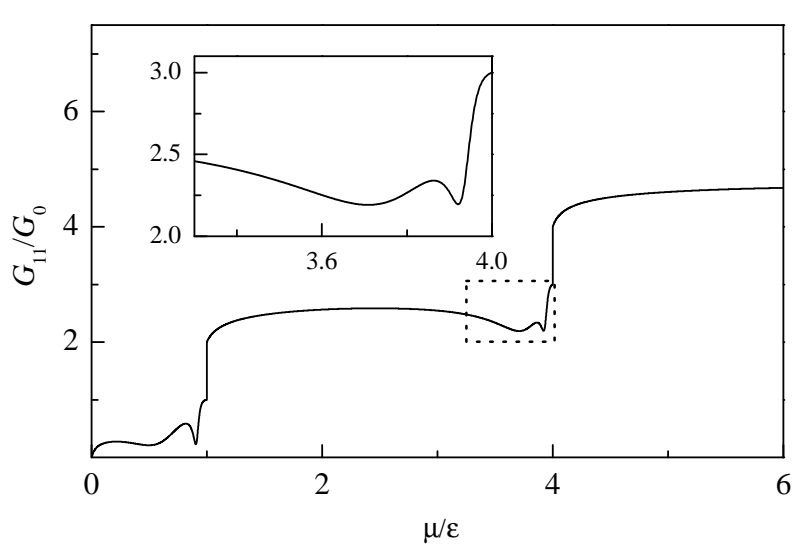

FIG. 4: Conductance $G_{11}$ versus the Fermi energy $\mu$ at $r_{1}=$ $r_{2}, \lambda_{1}=\lambda_{2}=4 r_{1}$, and $a=4$. The inset represents a fragment of the curve marked with the dotted rectangle.

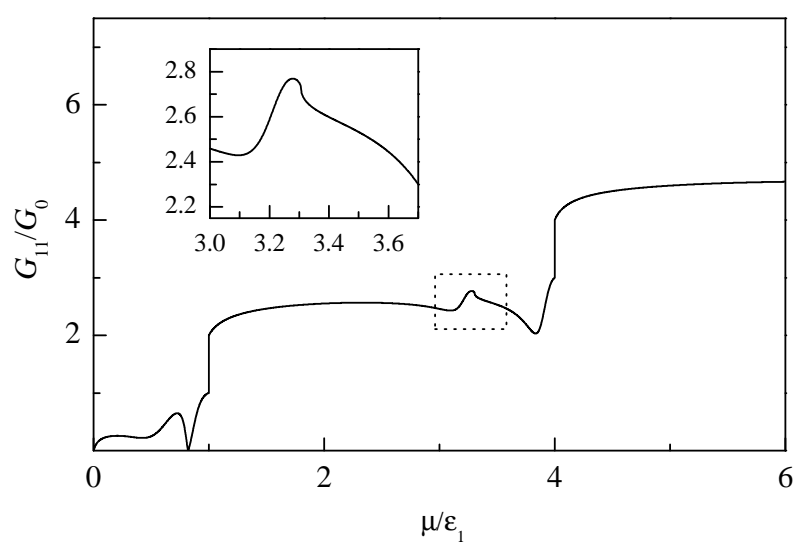

FIG. 5: Conductance $G_{11}$ versus the Fermi energy $\mu$ at $r_{2}=$ $1.1 r_{1}, \lambda_{j}=4 r_{j}$, and $a=4$. The inset represents a fragment of the curve marked with the dotted rectangle.

that $\widetilde{Q}_{j}(E)$ has a root singularity at the point $E_{m}^{(j)}$

$$
\widetilde{Q}_{j}(E)= \begin{cases}2 \sqrt{\frac{\varepsilon_{j}}{E_{m}^{(j)}-E}}+F(E), & E \rightarrow E_{m}^{(j)}-0, \\ 2 i \sqrt{\frac{\varepsilon_{j}}{E-E_{m}^{(j)}}}+F(E), & E \rightarrow E_{m}^{(j)}+0\end{cases}
$$

where $F(E)$ is a smooth function of $E$. It is worth mentioning that the root singularity similar to Eq. (19) is present in the electron density of states on the cylinder. It is convenient to represent $Q_{j}(E)$ in the form $Q_{j}(E)=\left|Q_{j}(E)\right| \exp \left(i \phi_{j}\right)$. One can see from Eq. (19) that the argument $\phi_{j}(E)$ changes abruptly by $\pi / 2$ at the point $E_{m}^{(2)}$. The behavior of the function $\phi_{j}(E)$ in the interval $\left(E_{m}^{(j)}, E_{m+1}^{(j)}\right)$ depends on the scattering length $\lambda_{j}$. In the case of the strong point potential $\left(\lambda_{j} \ll r\right)$ the argument $\phi_{j}(E)$ decreases monotonically from $\pi / 2$ to zero when the energy varies from $E_{m}^{(j)}$ to $E_{m+1}^{(j)}$. In the case of the large scattering length $\left(\lambda_{j} \gg r\right)$ the argument $\phi_{j}$ has a maximum $\phi_{j}^{\max }$ in the range $\left(\pi / 2<\phi_{j}^{\max }<\pi\right)$.
Using Eq. (19), we obtain the following asymptotics for $G_{11}(\mu)$ in the vicinity of $E_{m}^{(2)}$ :

$$
G_{11}(\mu) \simeq G_{1}(\mu)+G_{2}(\mu),
$$

where $G_{1}(\mu)$ is given by

$$
G_{1}=G_{0}\left(N(\mu)-\frac{\left(\operatorname{Im} \widetilde{Q}_{1}\right)^{2}}{\left|\widetilde{Q}_{1}\right|^{2}}\right)
$$

and $G_{2}(\mu)$ has the form

$$
G_{2}(\mu)=-\frac{G_{0}|a|^{2}}{\left|\widetilde{Q}_{1}\right|} \sqrt{\left|\mu-E_{m}^{(2)}\right|} \sin 2 \phi_{1} \sin \left(\phi_{1}+\phi_{2}\right)
$$

Here $G_{1}(\mu)$ is the conductance of the single cylinder with the point perturbation. This term is regular at the point $\mu=E_{m}^{(2)}$. The second term $G_{2}(\mu)$ in Eq. (20) is stipulated by the influence of the second tube. Eq. (22) shows that the derivative of $G_{2}(\mu)$ has the root singularity at the point $E_{m}^{(2)}$.

Two different line shapes are possible depending on the value of $\phi_{1}$. If $\phi_{1}<\pi / 2$, then $\sin \left(\phi_{1}+\phi_{2}\right)>0$ and $\sin \left(2 \phi_{1}\right)>0$ on both sides of $E_{m}^{(2)}$. Under these conditions, $G_{2}(\mu)$ is negative and reaches its maximal value $G_{2}=0$ at the point $E_{m}^{(2)}$. Hence, the dependence $G_{11}(\mu)$ has a sharp peak at the point $E_{m}^{(2)}$ (Fig. 6 6 ). If $\phi_{1}>\pi / 2$, then the sign of $G_{2}(\mu)$ is changed at $\mu=E_{m}^{(2)}$ and the curve $G_{11}(\mu)$ has the form represented in Fig.6 $b$.

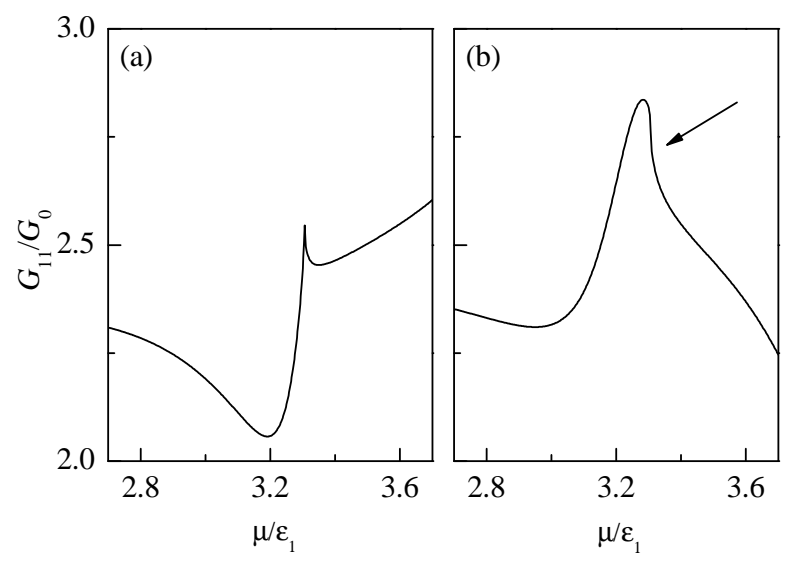

FIG. 6: Conductance $G_{11}$ versus the Fermi energy $\mu$ at $r_{2}=$ $1.1 r_{1}$ and $a=10$. (a) $\lambda_{1}=\lambda_{2}=0.3 r_{1}\left(\phi_{1}<\pi / 2\right)$; (b) $\lambda_{1}=\lambda_{2}=5 r_{1}\left(\phi_{1}>\pi / 2\right)$. The arrow marks the point $\mu=E_{m}^{(2)}$.

It is obvious that at a finite temperature the dependence $G_{11}(\mu)$ is smooth and the asymmetric peaks resemble Fano resonances. However, the origin of peaks and dips in the conductance of crossed cylinders differs from the origin of the Fano resonances. Fano resonances emerge from the coherent interaction of a discrete state 
and a continuum while the peaks and dips in the conductance $G_{11}(\mu)$ are stipulated by root singularities in the density of states.

The second term in Eq. (15) has a step down of amplitude $G_{0}$ at the point $\mu=E_{m}$. Hence, the amplitude of the conductance steps equals $G_{0}$ in contrast to the case of unperturbed cylinder where the amplitude equals $2 G_{0}$.

Let us consider now the conductance $G_{21}$ that corresponds to the transmission of electrons from the first cylinder to the second one. Conductance $G_{21}$ determines transport properties of the system in the case when the contacts $1 a$ and $1 b$ in Fig. 1 have equal potential $V_{1}$ while the contacts $2 a$ and $2 b$ have the potential $V_{2}$. There are

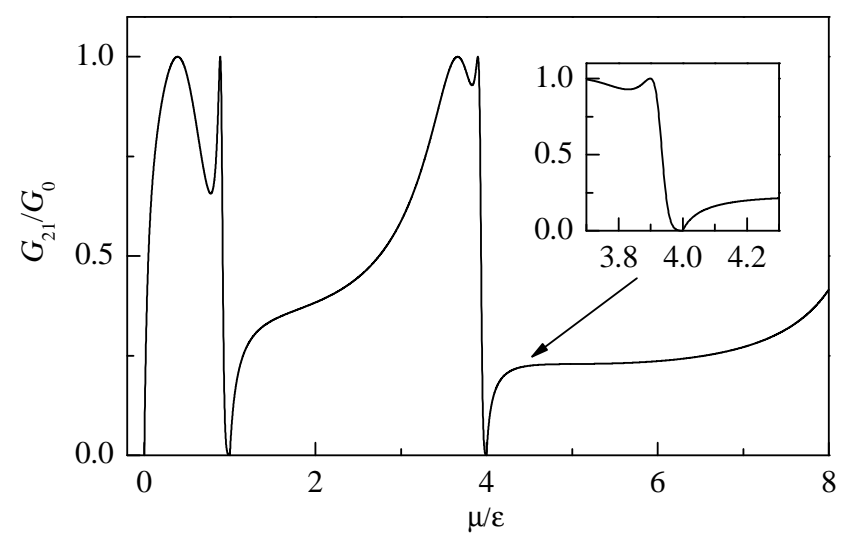

FIG. 7: Conductance $G_{21}$ versus the Fermi energy $\mu$ at $r_{1}=$ $r_{2}, \lambda_{1}=\lambda_{2}=3 r_{1}$, and $a=5$. The inset represents the same dependence in the vicinity of $\mu=4 \varepsilon$.

four ways for an electron to pass from the first tube to the second one: $1 a \rightarrow 2 a, 1 a \rightarrow 2 b, 1 b \rightarrow 2 a$, and $1 b \rightarrow 2 b$. Due to the symmetry of the system the transmission amplitudes of all the transitions are equal. The transmission amplitudes $t_{m^{\prime} m}^{21}$ are determined in the same way as $t_{m^{\prime} m}^{11}$

$$
t_{m^{\prime} m}^{21}(E)=\frac{i \widetilde{Q}_{2}}{\widetilde{Q}_{1} \widetilde{Q}_{2}-|a|^{2}} .
$$

Using the Landauer formula and Eq. (14), we obtain

$$
G_{21}(\mu)=4 G_{0} \frac{|a|^{2} \operatorname{Im} \widetilde{Q}_{1} \operatorname{Im} \widetilde{Q}_{2}}{\left.\left.\left|\widetilde{Q}_{1} \widetilde{Q}_{2}-\right| a\right|^{2}\right|^{2}} .
$$

Here the factor 4 is stipulated by four ways for the electron to propagate from reservoirs marked $1 a$ and $1 b$ in Fig. [1 to reservoirs marked $2 a$ and $2 b$. The dependence $G_{21}(\mu)$ is represented in Figs. 7. 9.

One can see from Eq. (24) that the conductance $G_{21}(\mu)$ vanishes when the Fermi energy $\mu$ coincides with $E_{m}^{(j)}$. Using Eq. (19), we get the following form for $G_{21}(\mu)$ in the vicinity of $E_{m}^{(j)}$ :

$$
G_{21}(\mu)= \begin{cases}A_{l}\left(E_{m}^{(j)}-\mu\right), & \mu \rightarrow E_{m}^{(j)}-0, \\ A_{r} \sqrt{\mu-E_{m}^{(j)}}, & \mu \rightarrow E_{m}^{(j)}+0 .\end{cases}
$$

where $A_{l}$ and $A_{r}$ are positive factors. One can see that the dependence $G_{21}(\mu)$ exhibits a sharp kink at the point $E_{m}^{(j)}$ (Fig. 7). The kink is stipulated by the root singularity in the density of states. Similar line shape had been found earlier in the conductance of the quantum cylinder with one-dimensional leads $\underline{\underline{49}}$ An asymmetric conductance peak appears in a vicinity of the point $\mu=E_{m}^{(j)}$. The form of the peak is similar to the Fano resonance line shape, however the dependence $G_{21}(\mu)$ at $T=0$ is not smooth in contrast to the Fano curve.

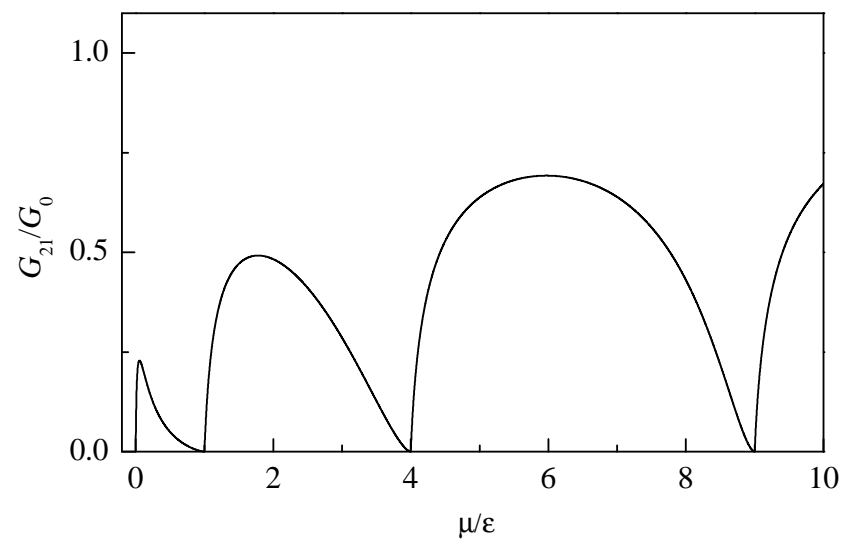

FIG. 8: Conductance $G_{21}$ versus the Fermi energy $\mu$ at $r_{1}=$ $r_{2}, \lambda_{1}=\lambda_{2}=0.1 r_{1}$, and $a=5$.

Let us determine the maximal value of the conductance $G_{21}(\mu)$. Using real variables $\xi_{j}=\operatorname{Re} \widetilde{Q}_{j} / \operatorname{Im} \widetilde{Q}_{j}$ and $\eta=|a|^{2} /\left(\operatorname{Im} \widetilde{Q}_{1} \operatorname{Im} \widetilde{Q}_{2}\right)$, we can express the conductance $G_{21}(\mu)$ in the following form:

$$
G_{21}\left(\xi_{1}, \xi_{2}, \eta\right)=4 G_{0} \frac{\eta}{\left(\xi_{1} \xi_{2}-\eta-1\right)^{2}+\left(\xi_{1}+\xi_{2}\right)^{2}} .
$$

If $\eta<1$, then $G_{21}$ as a function of $\xi_{1}$ and $\xi_{2}$ has only one maximum $G_{21}=4 G_{0} \eta /(\eta+1)^{2}$ at $\xi_{1}=\xi_{2}=0$. It is obvious, that $G_{21}<G_{0}$ in this case. The condition $\xi_{1}=\xi_{2}=0$ is equivalent to $\operatorname{Re} Q_{1}=\operatorname{Re} Q_{2}=0$. That is possible only in the case of identical tubes and large scattering lengths. In the opposite case of strong point perturbation $\left(\lambda_{j} \ll r_{j}\right)$ the function $\operatorname{Re} Q_{j}(E)$ has no zeros and the maxima of the conductance are situated near the minima of $\operatorname{Re} Q_{j}(E)$ (Fig. 9). In this limit, the maximal value of the conductance is smaller than $G_{0}$.

An additional maximum $G_{21}=G_{0}$ appears at $\xi_{1}=$ $\xi_{2}=\sqrt{\eta-1}$ in the case $\eta \geq 1$ (Fig. 77). That means the coupling between wave functions on different nanotubes is strong. The conductance $G_{21}$ decreases with decreasing of the coupling. Therefore, the conductance $G_{21}$ never exceeds $G_{0}$. We relate this result to limited transparency of the point contact. We stress that the result is not trivial because the sum in Landauer's equation (6) contains many terms. The condition $\xi_{1}=\xi_{2}$ is satisfied only if the tubes are identical. Hence, the conductance $G_{21}$ reaches its maximal possible value only in 


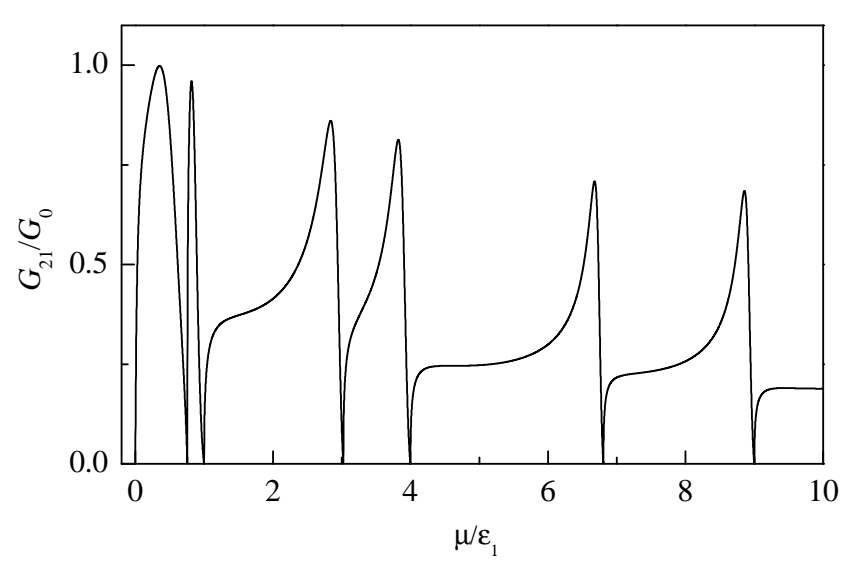

FIG. 9: Conductance $G_{21}$ versus the Fermi energy $\mu$ at $r_{2}=$ $1.15 r_{1}, \lambda_{1}=\lambda_{2}=5 r_{1}$, and $a=8$.

the case of identical tubes and strong coupling between the wave functions $(|a| \gtrsim 1)$.

If radii of tubes are different, then the number of zeros on the dependence $G_{21}(\mu)$ increases because $E_{m}^{(1)} \neq E_{m}^{(2)}$ (Fig. 9). The maximal value of the conductance $G_{21}$ is also smaller then $G_{0}$ in this case.

\section{CONCLUSION}

The electron transport in crossed conductive nanocylinders is investigated using the LandauerBüttiker formalism. An explicit form for the conductance $G_{11}$ as a function of the Fermi energy $\mu$ is obtained. We have shown that the conductance of each tube contains resonance dips stipulated by the back-scattering of electrons on the contact. The maximal value of dips does not exceed a unit of the conductance quantum. Positions of dips depend on parameters of the contact. In the case of identical tubes the conductance can exhibit no more than two dips on each plateau. If the radii of tubes are different, then the dependence of the conductance on the Fermi energy contains asymmetric peak-dip structures of the form given by Eq. (4). The dips and peaks are stipulated by the root singularities in the density of states. It is worth mentioning that the asymmetric peaks and dips have been observed experimentally in the conductance of many carbon nanotube based systems, in particular in crossed nanotubes $\stackrel{18}{=}$. The asymmetric line shapes were attributed to Fano resonances that emerge from the interference of the bound states with the continuum. Results of the present paper provide alternative possible explanation of the asymmetric peaks and dips. According to our results they might originate from the root singularities in the density of states.

The conductance $G_{21}$ which is related to transmission of electrons from the first cylinder to the second one has the resonance nature. The maximal value of the conductance $G_{21}$ is a unit of the conductance quantum. The condition of maximal transmission is satisfied only if the tubes are identical. Similar results have been obtained in Ref. 23 for several crossed junctions of small nanotubes. It should be noted, that the resistance of the value $16.8 \mathrm{k} \Omega \gtrsim G_{0}^{-1}$ has been observed experimentally ${ }^{18}$ for crossed carbon nanotubes at $T=4.2 \mathrm{~K}$.

The work is support by the Russian Foundation for Basic Research (grant No. 05-02-16145).
* Electronic address: pyataevma@math.mrsu.ru

1 W. Liang, M. Bockrath, D. Bozovici, J. H. Hafner, M. Tinkhami, H. Park, Nature (London) 411, 665 (2001).

2 J. Kong, E. Yenilmez, T. W. Tombler, W. Kim, H. Dai, R. B. Laughlin, L. Liu, C. S. Jayanthi, and S. Y. Wu, Phys. Rev. Lett. 87, 106801 (2001).

3 Z. Zhang and V. Chandrasekhar, Phys. Rev. B 73, 075421 (2006).

4 O. V. Kibis, D. G. W. Parfitt, and M. E. Portnoi, Phys. Rev. B 71, 035411 (2005).

${ }^{5}$ C. Kilic, S. Ciraci, O. Gülseren, and T. Yildirim, Phys. Rev. B 62, R16345 (2000).

6 M. P. Anantram and F. Léonard, Rep. Prog. Phys. 69, 507 (2006).

7 S. J. Tans, M. H. Devoret, H. Dai, A. Thess, R. Smalley L. J. Geerlings, and C. Dekker, Nature (London) 386, 474 (1997).

8 O. V. Kibis and M. E. Portnoi, Pis'ma Zh. Tekh. Fiz. 31, No. 15, 85 (2005) [Tech. Phys. Lett. 31, No. 8, 671 (2005)].

9 S. J. Tans, A. R. M. Verschueren, and C. Dekker, Nature (London) 393, 49 (1998).

10 R. Martel, T. Schmidt, H. R. Shea, T. Hertel, and Ph. Avouris, Appl. Phys. Lett. 73, 2447 (1998).
11 H. W. Ch. Postma, T. Teepen, Z. Yao, M. Grifoni, and C. Dekker, Science 293, 76 (2001).

12 A. Bachtold, P. Hadley, T. Nakanishi, and C. Dekker, Science 294, 1317 (2001).

13 J. Appenzeller, J. Knoch, M. Radosavljevic, and Ph. Avouris, Phys. Rev. Lett. 92, 226802 (2004).

14 Z. Yao, H. W. Ch. Postma, L. Balents, and C. Dekker, Nature (London) 402, 273 (1999).

15 C. Zhou, J. Kong, E. Yenilmez, and H. Dai, Science 290, 1552 (2000).

16 J. U. Lee, P. P. Gipp, and C. M. Heller, Appl. Phys. Lett. 85, 145 (2004).

17 M. S. Fuhrer, J. Nygard, L. Shih, M. Forero, Y.-G. Yoon, M. S. C. Mazzoni, H. J. Choi, J. Ihm, S. G. Louie, A. Zettl, and P. L. McEuen, Science 288, 494 (2000).

18 J. Kim, J.-R. Kim, Jeong-O Lee, J. W. Park, H. M. So, N. Kim, K. Kang, K.-H. Yoo, and J.-J. Kim, Phys. Rev. Lett. 90, 166403 (2003).

19 B. Gao, A. Komnik, R. Egger, D. C. Glattli, and A. Bachtold, Phys. Rev. Lett. 92, 216804 (2004).

20 S. Heinze, J. Tersoff, R. Martel, V. Derycke, J. Appenzeller, and Ph. Avouris, Phys. Rev. Lett. 89, 106801 (2005). 
21 L. F. Chibotaru, S. Compernolle, and A. Ceulemans, Phys. Rev. B 68, 125412 (2003).

${ }^{22}$ F. Y. Meng, S. Q. Shi, D. S. Xu, and R. Yang, Phys. Rev. B 70, 125418 (2004).

23 S. Dag, R. T. Senger, and S. Ciraci, Phys. Rev. B 70, 205407 (2004).

24 G. Kim, S. B. Lee, T.-S. Kim, and J. Ihm, Phys. Rev. B 71, 205415 (2005).

${ }^{25}$ G. Kim, S. B. Lee, H. Lee, and J. Ihm, J. Phys.: Condens. Matter 19, 026217 (2007).

${ }^{26}$ H. T. Yang, J. W. Chen, L. F. Yang, and J. Dong, Phys. Rev. B 71, 073401 (2005).

27 M. del Valle, C. Tejedor, and G. Cuniberti, Phys. Rev. B 71, 125306 (2005).

28 U. Fano, Phys. Rev. 124, 1866 (1961).

29 J. U. Nöckel, Phys. Rev. B 46, 15348 (1992).

30 A. A. Clerk, X. Waintal, and P. W. Brouwer, Phys. Rev. Lett. 86, 4636 (2001).

31 C. S. Kim and A. M. Satanin, Zh. Exp. Teor. Fiz. 115, 211 (1999) [JETP 88, 118 (1999)].

32 C. S. Kim and A. M. Satanin, Y. S. Joe, R. M. Cosby, Phys. Rev. B 60, 10962 (1999).

33 C. S. Kim and A. M. Satanin, Physica E 4, 211 (1999).

34 C. S. Kim, O. N. Roznova, A. M. Satanin, and V. B. Stenberg, Zh. Exp. Teor. Fiz. 121, 1157 (2002) [JETP 94, 992 (2002)].

35 J. Göres, D. Goldhaber-Gordon, S. Heemeyer, M. A. Kastner, H. Shtrikman, D. Mahalu, and U. Meirav, Phys. Rev. B 62, 2188 (2000).

${ }^{36}$ K. Kobayashi, H. Aikawa, S. Katsumoto, and Y. Iye, Phys. Rev. Lett. 88, 256806 (2002).
37 B. Babič and C. Schönenberger, Phys. Rev. B 70, 195408 (2004).

38 A. V. Chaplik, L. I. Magarill, D. A. Romanov, Physica B 249-251, 377 (1998).

39 L. I. Magarill, D. A. Romanov, and A. V. Chaplik, Zh. Exp. Teor. Fiz. 113, 1411 (1998) [JETP 86, 771 (1998)].

${ }^{40}$ V. A. Margulis, A. V. Shorokhov, and M. P. Trushin, Phys. Lett. A 276, 180 (2000).

41 N. Nemec and G. Cuniberti, Phys. Rev. B 74, 165411 (2006).

42 A. A. Grigor'kin and S. M. Dunaevskiř, Phys. Solid State 49, 585 (2007).

43 V. Ya. Prinz, V. A. Seleznev, A. K. Gutakovsky, A. V. Chehovskiy, V. V. Preobrazhenskii, M. A. Putyato, and T. A. Gavrilova, Physica E 6, 828 (2000).

44 A. B. Vorob'ev V. Ya. Prinz, Yu. S. Yukecheva, and A. I. Toropov, Physica E 23, 171 (2004).

${ }^{45}$ Y. N. Demkov and V. N. Ostrovsky, Zero-Range Potentials and Their Applications in Atomic Physics (Plenum, New York, 1988).

46 S. Albeverio, F. Gesztesy, R. Hegh-Krohn, and H. Holden, Solvable Models in Quantum Mechanics (Springer-Verlag, Berlin, 1988).

47 J. Brüning and V. A. Geyler, J. Math. Phys. 44, 371 (2003).

48 V. A. Geyler, V. A. Margulis, and M. A. Pyataev, Zh. Exp. Teor. Fiz. 124, 851 (2003) [JETP 97, 763 (2003)].

${ }^{49}$ V. A. Margulis and M. A. Pyataev, Phys. Rev. B 72, 075312 (2005). 